could have been a mathematician and Bernstein the listener which would have given him a chance to show more directly where his understanding breaks lecture rather than seeing the kind of effort it takes to understand the complexities of metamathematics.

But the long piece on US physicist I. I. Rabi is a gem. This is Bernstein at his best. We have a fascinating story of the shift of the centre of gravity of physics research from Europe to the US in the inter-War a Nobel Prizewinner in 1944 for work on molecular beams. Bernstein succeeds in presenting a fresh account of the most romantic period in the history of physics. Here is Rabi criticising the down. Instead we end up hearing a years as seen through the eyes of Rabi,

present division of labour between theorists and experimentalists in physics: "You do an experiment because your own philosophy makes you want to know the result. It's too hard, and life is too short, to spend your life doing something because someone else has said it's important. You must feel the thing yourself-feel that it will change your outlook and your way of life. You must bring it back to the human condition, the human expression - much closer to what the artist is supposed to feel." In the present period there are not many physicists who can experience this kind of emotional commitment to their research.

Joe Schwartz is on leave from the City University of New York.

\section{Gravitation and the expansion of the Earth}

\section{T. C. Van Flandern}

Cosmology and Geophysics. By P. S. Wesson. Pp. 240. (Adam Hilger: Bristol, UK, 1978.) $£ 17.50$.

IT is not immediately evident that the book's two subjects, cosmology and geophysics, have very much to do with each other. The primary connection is through expansion of the Earth, a predicted consequence of several recently proposed cosmological theories and a favourite theme of the author. If the Earth does indeed expand with time, this provides a driving mechanism for continental drift, a simple understanding of how the Earth's surface became partitioned into continents and ocean basins, and in general a deeper understanding of the origin and evolution of the surface features of planetary bodies. The evidence that the Earth has, in fact, been expanding in the past is still debated; but the author presents a credible case for his interpretation. The evidence ranges from the suggestion that all of the continents would fit together nicely on a globe having one-fourth the Earth's present surface area, to palaeomagnetic determinations of the Permian radius, and arguments from logic: How could Antarctica become almost surrounded by an ocean ridge, with which it was once supposedly in contact, unless the globe has expanded?

Two mechanisms for producing the expansion are offered, and this leads us immediately into cosmology. The first is that the universal gravitational constant may be decreasing; the second is an expansion of all macroscopic bodies and the space they occupy relative to the size of the atomic particles which comprise them, at the same rate as the observed large scale expansion of the Universe. These two mechanisms can now be unified into a single theory, which has been the direction that most theoretical work has taken since this book was written.

The book opens with an excellent survey of variable- $G$ cosmologies and what motivated them; Milne, Dirac, Jordan, Gilbert, Brans and Dicke, and Hoyle and Narlikar are all reviewed. There follows a general discussion of post-Einstein gravitational theories, of the various attempts to incorporate Mach's Principle of Inertia into these theories, of space variability of the gravitational constant, vacuum elasticity, possible anisotropy of gravitation and the limits placed by the Eötvös experiment, and additional related topics. There is then an entire chapter devoted to the Eddington numbers, which form the basis of the Large Numbers Hypothesis and the motivation for the original 1937 proposal of Dirac that gravitation must be weakening with time. This chapter also discusses experimental limits on the variations of other fundamental quantities, such as the fine structure constant, and discusses the physical and astrophysical consequences of the proposed changes in fundamental constants of physics. book. It is a truly excellent review with a very complete bibliography. Moreover I was pleased to see explicit mention of the assumption underlying this entire discussion, that there may be as yet undiscovered laws of physics. This
This summarises the first half of the has always seemed to me an obvious point as long as our understanding of the true nature of phenomena such as gravitation is so limited (Why do masses attract only? Is there a quantum of gravitation? Is shielding possible?); yet it is an assumption few physicists seem to make. The only criticism to this point is that reading would not be easy going for the novice, as specialised terms are sometimes introduced with only very sketchy definitions and no real attempt to provide understanding of the concepts. There is also confusion on p42 about just what information ancient eclipses provide.

The second half of the book deals with experimental evidence on changes in gravitation and Earth expansion, and an attempted reconciliation of such concepts with Relativity Theory. The author very much favours the idea that the Earth has nearly doubled its radius in its lifetime, rather than the more modest expansion of perhaps $10 \%$ allowed by a weakening of gravity. I think he has overlooked a valuable argument. We know that the Moon must have been quite close to the Earth in the past; the best estimates place the closest approach at about $3 \times 10^{9} \mathrm{yr}$ ago. Tidal friction under these circumstances must have been enormous, and surely must have produced considerable internal heating and expansion of the Earth. Moreover, if the Earth had a much smaller radius than at present for most of this early period, this would help explain why the tidal coupling coefficient has averaged less than half its present value since the Moon was close. Whether the expansion continues today would then depend on whether gravitation is weakening; but the bulk of the expansion would have taken place early in the Earth's history. Moreover, it is important to keep in mind that expansion from any cause would surely occur in discrete events rather than continuously, as radial strain, like horizontal strain, would have to reach a threshhold before the Earth would yield. It is interesting to note the recent discovery of a huge surface fissure on Venus, comparable to the largest known on Mars. Taken with the large Van de Graff lunar farside surface depression, it is probably not premature to infer that expansion may indeed be a regular feature of planetary evolution. If so, Wesson deserves much of the credit for advocating the idea while it was still unpopular, and for placing it in a reasonable theoretical and observational perspective.

T. C. Van Flandern is Chief of the Celestial Mechanics Branch of the Nautical Almanac Office, US Naval Observatory, Washington, $D C$. 